\title{
Spiking Activity of a LIF Neuron in Distributed Delay Framework
}

\author{
Saket Kumar Choudhary, Karan Singh, *Vijender Kumar Solanki
}

\author{
Anna University, Chennai, Tamilnadu, India
}

\begin{abstract}
Evolution of membrane potential and spiking activity for a single leaky integrate-and-fire (LIF) neuron in distributed delay framework (DDF) is investigated. DDF provides a mechanism to incorporate memory element in terms of delay (kernel) function into a single neuron models. This investigation includes LIF neuron model with two different kinds of delay kernel functions, namely, gamma distributed delay kernel function and hypo-exponential distributed delay kernel function. Evolution of membrane potential for considered models is studied in terms of stationary state probability distribution (SPD). Stationary state probability distribution of membrane potential (SPDV) for considered neuron models are found asymptotically similar which is Gaussian distributed. In order to investigate the effect of membrane potential delay, rate code scheme for neuronal information processing is applied. Firing rate and Fano-factor for considered neuron models are calculated and standard LIF model is used for comparative study. It is noticed that distributed delay increases the spiking activity of a neuron. Increase in spiking activity of neuron in DDF is larger for hypo-exponential distributed delay function than gamma distributed delay function. Moreover, in case of hypo-exponential delay function, a LIF neuron generates spikes with Fano-factor less than 1.
\end{abstract}

Keywords - Distributed Delay Framework, Fokker-Planck Equation, Gamma Distribution, Hypo-exponential Distribution, Stationary Probability Distribution, Spiking Activity.

\section{INTRODUCTION}

$\mathrm{T}$ HERE are a number of neuron models depending on biophysical and electrical properties of a neuron suggested in literature [1-5]. Among these neuron models, Leaky integrate-and-fire (LIF) model has become a backbone for theoretical as well as experimental investigation of neuronal dynamics due to its simplicity and analytical solvable capability $[5,6,7]$. This model is an RC-circuit equivalent representation of a neuron with additional spiking constraint and is widely used for mathematical explanation of bio-physical mechanism and information processing of neurons $[1,2,4,8,9]$. Based on some specific properties, many variants of LIF model are suggested in literature $[1,5,7,8]$. These neuron models explain neuronal dynamics adequately, but when we talk about memory, we have to rely on group of few neurons or neural networks. None of these single neuron models capture memory element. Recently, Karmeshu et. al. [10] have suggested a distributed delay framework for incorporating the effect of previous values of membrane potential (memory) on neuronal dynamics, in which, a kernel function is included in LIF model to capture the aggregate effect of previous values of membrane potential on its further evolution. It is a challenging problem to find an appropriate kernel function so that the resulting model can explain most of the variability in neuronal responses. To this end, Karmeshu et. al. [10] have investigated their proposed framework with exponential distributed delay kernel and noticed exponential inter-spike-interval (ISI) distribution, whereas Sharma and Karmeshu [11] have obtained transient bi-modality in ISI distribution for gamma distributed delay kernel. Choudhary et. al. [12] have studied LIF model in DDF with hypo-exponential distributed delay kernel and observed huge variability in spiking pattern of a LIF neuron as obtained in empirical data. These investigations [10, 11, 12] reflect the novelty and robustness of distributed delay framework for neuronal dynamics, but none of them suggests about the actual effect of distributed delay (memory) on membrane potential evolution and spiking activity of a neuron.

Ermentrout and Terman [2], Rudolph and Destexhe [13] have suggested methods for estimating the moments of excitatory and inhibitory conductances from stationary probability distribution of membrane potential (SPDV) for a neuron. Ruan and Filfil [14] have suggested about stability of steady state distribution of membrane potential for discrete delay case and calculated the critical values of discrete delay for stable steady state distribution of membrane potential. We are interested in investing the effect of memory element on evolution of membrane potential and spiking activity of a neuron in distributed delay framework [10]. To achieve the objectives, we have investigated the LIF model in DDF with two different memory kernel functions namely; (i) gamma distributed function (ii) hypo-exponential distributed function, and compared the findings with standard LIF neuron with stochastic input stimulus. SPDV for considered model is calculated in sub-threshold regime of a neuron, whereas, to notice the effect of memory kernel on neuronal information processing, firing rate of neuron and Fano-factor corresponding to spike-sequences is obtained.

The present article is divided in mainly five sections. After a brief discussion about literature survey and objective of article in Section 1, a massive discussion about DDF for a neuron is discussed in Section 2. This section also contains mathematical formulation of LIF model with gamma distributed kernel function and hypo-exponential distributed kernel function. Section 3 deals with SPDV of considered neuron models. Simulation based investigation for spiking activity of LIF neuron in DDF and their result interpretation is given in Section 4. Section 5 contains analysis of findings and conclusion.

\section{Distribution Delay Framework of a Neuron}

Generalized form for describing the rate of change of membrane potential reads $[2,8]$

$$
\frac{d V}{d t}=f(V, t)+I
$$

where $I$ is applied input stimulus and $f(V, t)$ is membrane potential-current relationship functions. Choices of $f(V, t)$ result into different kind of neuron models. The simplest representation of 
Eq. (1) is the LIF model which can be obtained by substitution of the $-\beta V$ for $f(V, t)$ LIF model with stochastic input stimulus is given as:

$$
\frac{d V}{d t}=-\beta V(t)+\mu+\xi(t)
$$

where $V(t)$ is membrane potential, $\beta$ is membrane decay constant, $\mu$ is mean input stimulus, $\xi(t)$ is delta correlated Gaussian white noise with intensity $\sigma$, i.e. $\left\langle\xi(t)>=0\right.$ and $<\xi\left(t_{i}\right) \xi\left(t_{j}\right)>=\frac{\sigma^{2}}{2}$.

Karmeshu et. al. [10] assumed that the evolution of membrane potential not only depends on applied input stimulus but also on its previous values (memory) and proposed a distributed delay framework to investigate neuronal dynamics. Memory kernel function

$\int_{0}^{t} K(t-\tau) V(\tau) d \tau$ is substituted for function $f(V, t)$ in Eq. (1) in DDF [10]. LIF model with distributed delay function $K(t)$ takes the form:

$$
\frac{d V(t)}{d t}=-\beta \int_{0}^{t} K(t-\tau) V(t) d \tau+\mu+\xi(t)
$$

Karmeshu et. al. [10], Sharma and Karmeshu [11] have investigated this DDF model in the presence gamma distributed delay kernel. Choudhary et. al. [12] have studied LIF model incorporating hypoexponential distributed delay kernel in DDF. Mathematical formulations of these studies [10-12] are described in following subsections.

\section{A. LIF Model with Gamma Distributed Delay Function}

Following Karmeshu et. al. [10], LIF model including gamma distributed delay reads as:

$$
\frac{d V}{d t}=-\beta \int_{0}^{t} \frac{\eta^{m+1}(t-\tau)^{m} e^{-\eta(t-\tau)}}{m !} V(\tau) d \tau+\mu+\xi(t)
$$

where $\eta$ and $m$ are delay and shape parameters, respectively. For $m=0$ Eq. (4) results neuron model with weak delay, which also a termed as neuron model with exponential distributed delay function, whereas, for $m \geq 1 \mathrm{Eq}$. (4) results neuron model with strong delay $[10,11]$. Incorporation of memory kernel in LIF model results a non-Markovian membrane potential process $\{V(t) ; t \geq 0\}$ which can be transformed into Markovian process in extended space [10].

Substitution of $U_{m}(t)=\int_{0}^{t} \frac{\eta^{m+1}(t-\tau)^{m} e^{-\eta(t-\tau)}}{m !} V(\tau) d \tau$ into Eq.

(4) and further simplification results a system of coupled SDE

$$
\begin{aligned}
& \frac{d V}{d t}=-\eta \beta U_{m}(t)+\mu+\xi(t) \\
& \frac{d U_{i}(t)}{d t}=-\eta\left\{U_{i}(t)-U_{i-1}(t)\right\} \\
& \frac{d U_{0}(t)}{d t}=-\eta\left\{U_{0}(t)-\eta V(t)\right\}
\end{aligned}
$$

for $i \in\{1,2, \ldots, m\}$, with initial condition $\quad[V(t)=0$ and $U_{j}(t)=0 \forall j \in\{0,1,2, \ldots, m\}$ at $\left.t=0\right]$.

\section{B. LIF with Hypo-Exponential Distributed Delay Function}

Sum of synaptic excitatory and inhibitory input stimulus at spikegenerator-locus (SGL) in soma determines the spike generation in a neuron [15]. These excitatory and inhibitory input stimulus result excitatory and inhibitory membrane potential, respectively [16]. Choudhary et. al. [12] has applied hypo-exponential distributed kernel function to incorporate the SGL mechanism in DDF. Following Choudhary et. al. [12], LIF model with hypo-exponential distributed delay function becomes

$$
\frac{d V}{d t}=-\frac{\beta \lambda_{E} \lambda_{I}}{\lambda_{E}-\lambda_{I}} \int_{0}^{t}\left(e^{-\lambda_{E}(t-\tau)}-e^{-\lambda_{I}(t-\tau)}\right) V(\tau) d \tau+\mu+\xi(t)
$$

with initial condition $V(t)$ at $t=0$, where $\lambda_{E}$ and $\lambda_{I}$ are arrival rate of excitatory membrane potential and inhibitory membrane potential.

In extended space, substitution of $U_{1}(t)=\int_{0}^{t} e^{-\lambda_{E}(t-\tau)} V(\tau) d \tau$ and $U_{2}(t)=\int_{0}^{t} e^{-\lambda_{I}(t-\tau)} V(\tau) d \tau$ into Eq. (6), model takes the form [12]

$$
\begin{aligned}
& \frac{d V}{d t}=-\frac{\beta \lambda_{E} \lambda_{I}}{\lambda_{I}-\lambda_{E}}\left(U_{1}-U_{2}\right)+\mu+\xi(t) \\
& \frac{d U_{1}(t)}{d t}=-\lambda_{E} U_{1}+V \\
& \frac{d U_{2}(t)}{d t}=-\lambda_{I} U_{2}+V
\end{aligned}
$$

with initial condition $V(t)=U_{1}(t)=U_{2}(t)=0$ at $t=0$.

\section{Stationary Probability Distribution of Membrane Potential (SPDV)}

SPDV is an interesting feature in theoretical studies due to the easier experimental verification than other neuro-physiological features [17, 18]. Neuron models in DDF, represented as in Eq. (5) and Eq. (7), are systems of coupled stochastic differential equations. Calculation of probability distribution of membrane potential requires corresponding Fokker-Planck equation. Fokker-Planck equation plays a vital role in investigation of stochastic formulation of a neuron $[6,19,20]$. Fokker-Planck equations are helpful in solving the first passage time problem, calculation of firing rate of neuron and in estimation of moments of stochastic conductance. Solution of Fokker-Planck equations depends on initial and boundary conditions in a great extent [20]. There are many boundary conditions, but two of them are more useful in neuronal model context, (i) absorbing boundary (ii) reflecting boundary [2]. When membrane potential of neuron reaches at boundary (threshold value), immediately a spike is generated with the condition $p\left(V^{t h}, t\right)=1$, in former boundary case, whereas in later case, probability flux $J(V, t)$ remains equal to zero, i.e. membrane potential of neuron never reaches at its firing threshold. We consider the reflecting boundary conditions to calculate the stationary probability distribution of membrane potential in different cases given below. 


\section{A. Neuronal Model Gamma Distributed Memory Kernel}

Let $p^{G}\left(V, U_{0}, U_{1}, \ldots, U_{m}, t\right)$ be the spatial probability distribution of membrane potential for LIF model with gamma distributed delay kernel. Fokker-Planck equation associated with Eq. (5) is

$$
\begin{aligned}
& \frac{\partial p^{G}}{d t}=\frac{\partial}{\partial V}\left\{\eta \beta U_{m}-\mu\right\} p^{G}+\prod_{i=1}^{m} \frac{\partial}{\partial U_{i}} \eta\left(U_{i}-U_{i-1}\right) p^{G} \\
& +\frac{\partial}{\partial U_{0}} \eta\left(U_{0}-V\right) p^{G}+\frac{\sigma^{2}}{2} \frac{\partial^{2} p^{G}}{\partial V^{2}}
\end{aligned}
$$

with boundary conditions:

$$
p^{G}\left(V, U_{0}, U_{1}, \ldots, U_{m}, t \mid t=0\right)=\delta\left(t-t_{0}\right) \prod_{i=0}^{m} \delta\left(u_{i}(t)-U_{i}\left(t_{0}\right)\right)
$$

and

$$
p^{G}\left(V, U_{0}, U_{1}, \ldots, U_{m}, 0\right)=V p^{G}\left(V, U_{0}, U_{1}, \ldots, U_{m}, t \mid t=0\right)=0
$$
as $V \rightarrow \infty$.

Define a differential operator $\nabla=\left(\frac{\partial}{\partial V} \frac{\partial}{\partial U_{m}} \ldots \frac{\partial}{\partial U_{0}}\right)$ as a row vector, and then Eq. (8) can be written as

$$
\frac{d p^{G}}{d t}=\nabla\left(A_{G}+\left(\nabla\left(B_{G} p^{G}\right)\right)^{T}\right)
$$

where

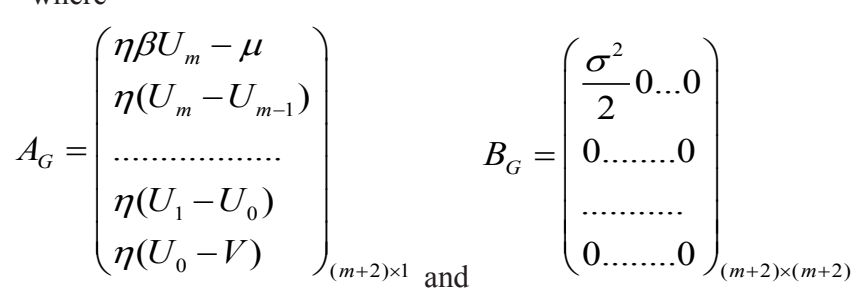

Therefore, probability current flux for gamma distributed kernel becomes

$$
J=\nabla\left(A_{G} p^{G}+\left(\nabla\left(B_{G} p^{G}\right)\right)^{T}\right)
$$

For stationary probability distribution $\frac{\partial p^{G}}{\partial t}=0, p^{G}=p_{S}{ }^{G}$ and
reflecting boundaries, Eq. (10) gives

$$
A_{G} p^{G}+\left(\nabla\left(B_{G} P^{G}\right)\right)^{T}=0
$$

Substituting the values of $A_{G}, B_{G}$ and using matrix calculation in Eq. (11), after simplification, we get

$$
\frac{1}{p_{S}{ }^{G}} \frac{\partial p_{S}{ }^{G}}{\partial V}=-\frac{\sigma^{2}}{2}(\beta V-\mu)
$$

Integration of Eq. (12) yields stationary probability distribution

$$
p_{S}{ }^{G}=k_{G} \exp \left\{-\frac{\beta}{\sigma^{2}}\left\{(V-\mu)^{2}-\mu^{2}\right\}\right\}
$$

\section{B. Neuronal Model Hypo-Exponential Distributed Memory Kernel}

Let $p H\left(V, U_{1}, U_{2}, t\right)$ be the spatial probability distribution of membrane potential for LIF model with hypo-exponential distributed delay. Fokker-Planck equation associated with Eq. (7) becomes

$$
\begin{aligned}
& \frac{\partial p^{H}}{d t}=\frac{\partial}{\partial V}\left\{\beta\left(U_{1}-U_{2}\right)-\mu\right\} p^{H}+\frac{\partial}{\partial U_{1}} \eta\left(\lambda_{E} U_{1}\right. \\
& -V) p^{H}+\frac{\partial}{\partial U_{2}} \eta\left(\lambda_{I} U_{2}-V\right) p^{H}+\frac{\sigma^{2}}{2} \frac{\partial^{2} p^{H}}{\partial V^{2}}
\end{aligned}
$$

with boundary conditions:

$$
\begin{gathered}
p^{H}\left(V, U_{1}, U_{2}, t \mid t=0\right)=\delta\left(t-t_{0}\right) \delta\left(U_{1}(t)-U_{1}\left(t_{0}\right)\right) \\
\delta\left(U_{2}(t)-U_{2}\left(t_{0}\right)\right) \text { and } \\
p^{H}\left(V, U_{1}, U_{2}, 0\right)=V p^{H}\left(V, U_{1}, U_{2}, t \mid t=0\right)=0 \text { as } V \rightarrow \infty .
\end{gathered}
$$

Define a differential operator $\nabla=\left(\frac{\partial}{\partial V} \frac{\partial}{\partial U_{1}} \frac{\partial}{\partial U_{2}}\right)$ as a row vector, and then Eq. (14) can be written as

$$
\frac{d p^{H}}{d t}=\nabla\left(A_{H}+\left(\nabla\left(B_{H} p^{H}\right)\right)^{T}\right)
$$

where $A_{H}=\left(\begin{array}{l}\beta\left(U_{1}-U_{2}\right)-\mu \\ \eta\left(\lambda_{E} U_{1}-V\right) \\ \eta\left(\lambda_{I} U_{2}-V\right)\end{array}\right)$ and $B H=\left(\begin{array}{rrr}\frac{\sigma^{2}}{2} & 0 & 0 \\ 0 & 0 & 0 \\ 0 & 0 & 0\end{array}\right)$.

Therefore, probability current flux becomes

$$
J=\nabla\left(A_{H}+\left(\nabla\left(B_{H} p^{H}\right)\right)^{T}\right)
$$

For stationary probability distribution $\frac{\partial p^{H}}{\partial t}=0, p^{H}=p_{S}{ }^{H}$ and for reflecting boundaries, Eq. (10) gives $\partial t$

$$
A_{H} p^{H}+\left(\nabla\left(B_{H} p^{H}\right)\right)^{T}=0
$$

Substituting the values of $A_{H}, B_{H}$ and using matrix calculation in Eq. (17), after simplification, we get

$$
\frac{1}{p_{S}{ }^{H}} \frac{\partial p_{S}{ }^{H}}{\partial V}=-\frac{\sigma^{2}}{2}(\beta V-\mu)
$$

Integration of Eq. (18) yields stationary probability distribution

$$
p_{S}{ }^{H}=k_{H} \exp \left\{-\frac{\beta}{\sigma^{2}}\left\{(V-\mu)^{2}-\mu^{2}\right\}\right\}
$$

Where $k_{H}$ is normalization constant.

Where $k_{G}$ is normalization constant. 


\section{LIF Model}

Let $p^{L}(V, t)$ be the spatial probability distribution of membrane potential for LIF model. Following Frank [20] and Burkitt [6, 19], Fokker-Planck equation associated with Eq. (2) is

$$
\frac{\partial p^{L}}{d t}=\frac{\partial}{\partial V}\{\beta V-\mu\} p^{L}+\frac{\sigma^{2}}{2} \frac{\partial^{2} p^{L}}{\partial V^{2}}
$$

with boundary conditions: $p^{L}(V, t \mid t=0)=\delta\left(t-t_{0}\right)$ and $p^{L}(V)=V p^{L}(V, t \mid t=0)=0$ as $V \rightarrow \infty$.

Probability current flux $(J)$ associated with Eq. (20) becomes

$$
J=(\beta V-\mu) p^{L}+\frac{\sigma^{2}}{2} \frac{\partial^{2} p^{L}}{\partial V^{2}}
$$

For stationary probability distribution $\frac{\partial p^{L}}{\partial t}=0, p^{L}=p_{S}{ }^{L}$ and for reflecting boundaries, Eq. (21) results

$$
\frac{1}{p_{S}{ }^{L}} \frac{\partial p_{S}{ }^{L}}{\partial V}=-\frac{\sigma^{2}}{2}(\beta V-\mu)
$$

Integration of Eq. (22) yields stationary probability distribution

$$
p_{S}{ }^{L}=k_{L} \exp \left\{-\frac{\beta}{\sigma^{2}}\left\{(V-\mu)^{2}-\mu^{2}\right\}\right\}
$$

where $k_{L}$ is normalization constant and can be calculated by using law of conservation of probabilities [19].

\section{Spiking Activity of a Neuron in DDF}

Spiking activity of a neuron exhibits a high level of variability [12, $21,22]$. This variability in spiking activity is essential for encoding information in form of spikes sequences [1,21,23]. Rate code scheme and temporal code scheme are two important strategies used by a neuron to encode information into spikes [3, 23-29]. Spike-count and Fano-factor related to the spike sequences are two important statistical measures of variability for rate code scheme $[22,29,30]$. Spike-count is used to encode information whereas Fano-factor provides a measure of variability in spike sequence, which can be computed as fallow [22, 29, 30].

Following Eden and Kramer [30], let spiking activity of a neuron is observed for $T$ time duration, and, if $t_{1}, t_{2}, t_{3}, \ldots$ be the spike time in observed spike sequence, divide time interval $[0, T]$ into $K$ subinterval of equal size $\delta(t)$ and count the number of spikes in each sub-interval. Let $n_{1}, n_{2}, \ldots, n_{K}$ be the number of spike in subintervals, respectively. Then

$$
\text { Mean Spike Count: } \stackrel{-}{N}=\frac{1}{K} \sum_{i=1}^{K} n_{i}
$$

Fano-factor for this spike-sequence can be computed as

$$
\text { Fano-factor: } F(\delta t)=\left(\frac{K}{K-1}\right) \frac{\sum_{i=1}^{K}\left(n_{i}-\bar{N}\right)^{2}}{\sum_{i=1}^{K} n_{i}}
$$

Fano-factor for a homogeneous Poisson process is exactly 1 [29], whereas, experimental data has Fano-factor distant from $1[4,5,17]$. In order to examine the effect of membrane potential delay on spiking activity and information processing of a neuron, we assume the rate code scheme of neuronal encoding and investigating the spike-count and the Fano-factor associated with spike sequence for considered neuron models. We apply Euler-Maruyama scheme in Monte-Carlo based simulation method [31, 32].

Let $\quad d X(t)=Y(X(t))+Z(X(t)) d W(t)$ be a stochastic differential equation, where $X, Y$ and $Z$ are state variables and $d W(t)$ is the Wiener process. Using Euler-Maruyama approximation in time sequences $\left\{t_{0}=0, t_{1}<, \ldots, t_{n}=t\right\}$, the considered SDE can be simulated as [32]

$$
X\left(t_{i+1}\right)=X\left(t_{i}\right)+Y\left(X\left(t_{i}\right)\right)\left(t_{i+1}-t_{i}\right)+Z\left(X\left(t_{i}\right)\right) \sqrt{t_{i+1}-t_{i}} w_{i+1}
$$

where $w_{1}, w_{2}, w_{3}, \ldots$ are independent normal variates and for fixed step size $(h): t_{i}=i h$, thus Eq. (26) takes the form

$$
X(i+1)=X(i)+Y(X(i)) h+Z(X(i)) \sqrt{h} w_{i+1}
$$

By applying above stated simulation strategy, we simulate neuron models given in Eqns. (2), (5) and (7). Eq. (5) is simulated for weak delay and strong delay, both, i.e. for $m=0$ and $m=1$. Spike-count and Fano-factor of our interest during simulation based study for various combination of parameter values are illustrated in Figs. 1-4 and in Figs. 5-7, respectively. In these Fig.s, legends SLIF, LIFG: $\mathrm{m}=0$, LIFG: $\mathrm{m}=1$ and LIFH are used which stand for simple LIF model, LIF model with gamma distributed delay having $m=0$, LIF model with gamma distributed delay having $m=1$ and LIF model with hypo-exponential distributed delay. Spike-count for neurons are calculated at fixed $\mu=0.1, \sigma=0.1$ and $\beta$ ranging from 0 to 0.25 with the step size of 0.01 , whereas, rest parameter values are given in Fig. caption. For $\beta=0$, considered neuron models becomes pure integrate-and-fire model, thus initial values of spike-count in Figs. 1-4 are same which is approximately about 100 spikes per second. This spiking activity of neurons decreases as $\beta$ increases, but, decrease in spiking activity in LIF models in DDF is smaller than simple LIF model due to presence of delay parameters. Critical value of delay parameters (i.e. $\eta=1, \lambda_{E}=1$ and $\lambda_{I}=1.01$ with input stimulus of small intensity) where all considered neuron models exhibit similar spiking activity is depicted in Fig. 1. When the value of $\eta, \lambda_{E}$ and $\beta$ is reduced, a neuron emits more spike as compared to simple LIF model due to dependency on past membrane potential values which works as memory element. LIFH neuron exhibits more spiking activity then other three neuron models due to the presence of delay in both

excitatory parameter $\left(\lambda_{E}\right)$ and inhibitory parameters $\left(\lambda_{I}\right)$, which is well illustrated in Fig.s 2-4. 


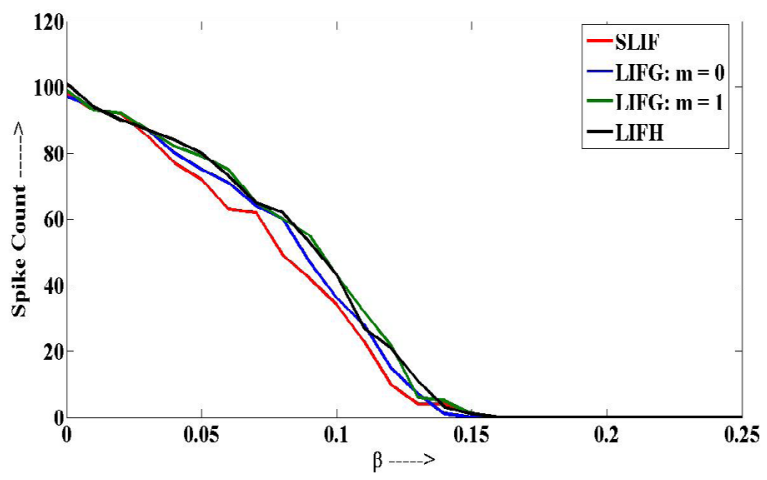

Fig. 1 Spike Count for $\eta=1, \lambda_{E}=1$ and $\lambda_{I}=1.01$.

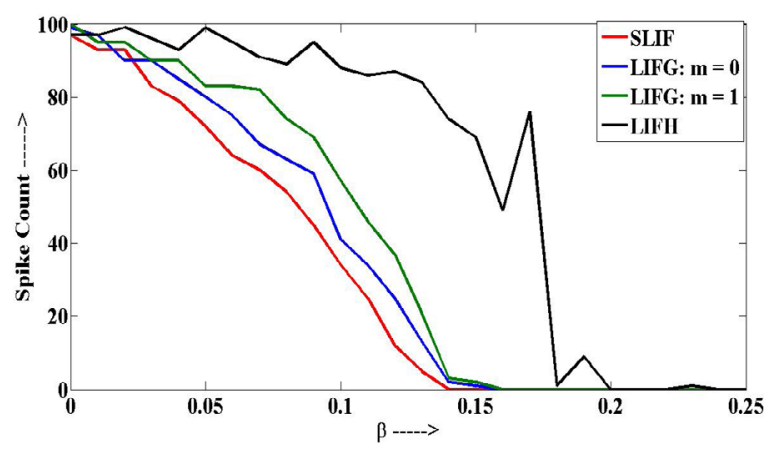

Fig. 2 Spike Count for $\eta=0.5, \lambda_{E}=0.5$ and $\lambda_{I}=1.01$.

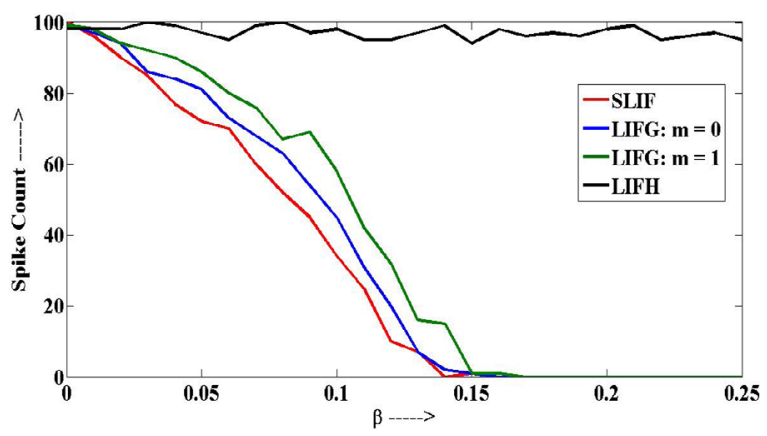

Fig. 3 Spike Count for $\eta=0.5, \lambda_{E}=1$ and $\lambda_{I}=0.1$.

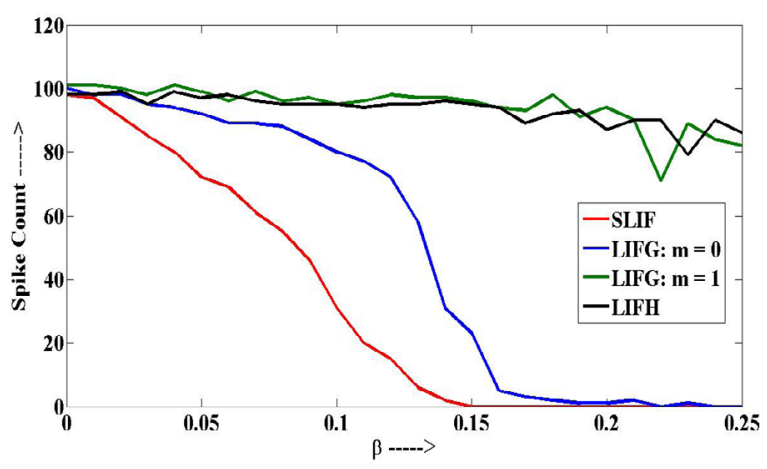

Fig. 4 Spike Count for $\eta=0.1, \lambda_{E}=0.1$ and $\lambda_{I}=0.11$.

These observations suggest that the spiking activity of a neuron increases in presence of memory kernel functions, and in this context, hypo-exponential distributed kernel function is more robust than gamma distributed kernel function. Now, a pertinent question arises that among these considered models, which neuron model is capable to generate the greater variability in its spiking activity. To this end, we have computed the Fano-factor of spike sequences obtained from simulation of considered neuron models.

Fano-factor (also known as index of dispersion) is defined as the ratio of variance to the mean number of spike generated by a neuron

in a fixed time period $\delta(t)$ which can be computed in many repetition of simulation of model with same input stimulus [5]. We have applied 100 times of repetition in our simulation based study for different combination of parameter values and corresponding results are illustrated in Figs. 5-7.

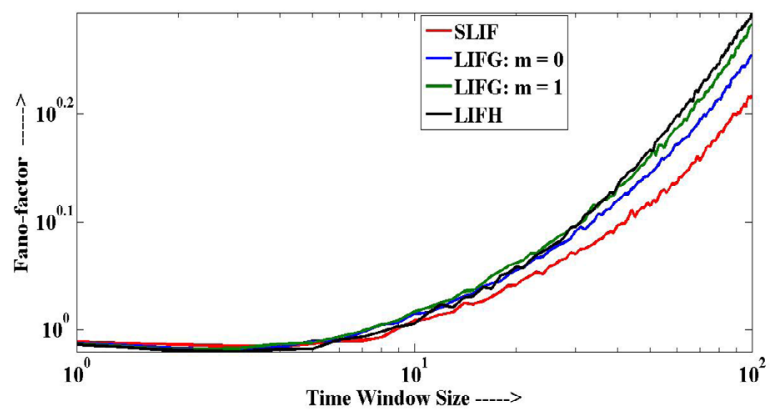

Fig. 5 Fano-factor for $\beta=0.1, \mu=0.1, \sigma=0.15, \eta=1, \lambda_{E}=1$ and $\lambda_{I}=0.99$.

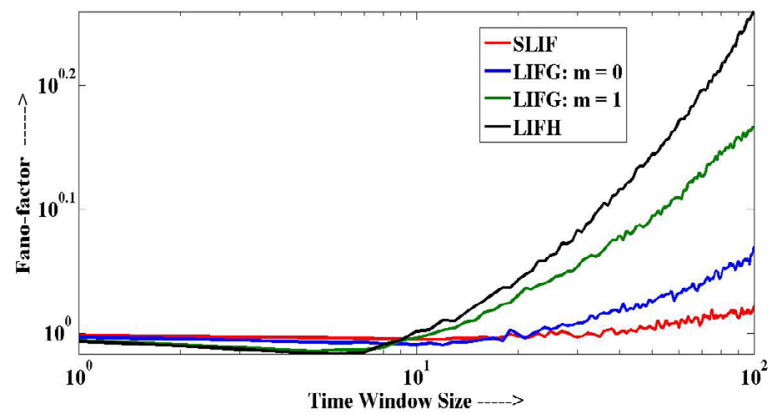

Fig. 6 Fano-factor for $\beta=0.25, \mu=0.2, \sigma=0.05, \eta=1$, $\lambda_{E}=1$ and $\lambda_{I}=0.9$

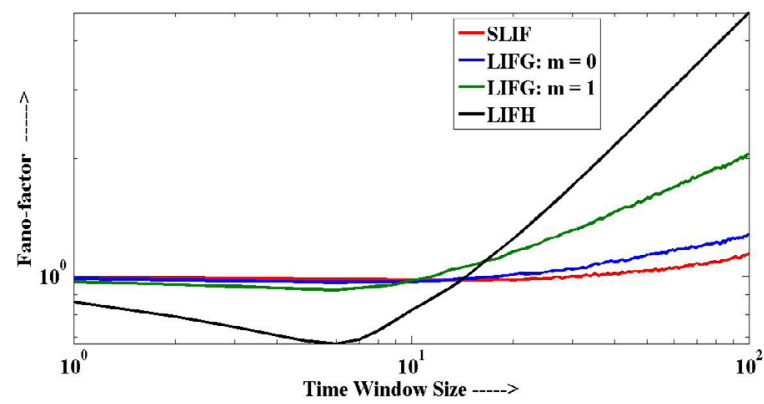

Fig. 7 Fano-factor for $\beta=0.25, \mu=0.2, \sigma=0.05, \eta=0.5$,

$\lambda_{E}=0.5$ and $\lambda_{I}=0.1$.

For critical parameter values where LIF model in DDF exhibits spiking activities similar simple LIF model, their Fano-factor also exhibits the similar behavior as illustrated in Fig. 5. It is shown in Fig. 5 and 6 that Fano-factor for considered neuron models are closer to 1 
for small time window, which is in a nice agreement with the Poisson hypothesis [5], whereas its value increases with respect to increase in time window. Increment in the Fano-factor is due to the long-term correlation in spiking activity [5], which is higher in LIF neuron with hypo-exponential distributed kernel function.

Various experimental studies have suggested that sensory systems exhibit the value of Fano-factor less than 1 [5]. We have noticed that when delay parameter values are smaller than critical values (as given in Fig. 1), LIF neuron with hypo-exponential kernel function is capable of generating spikes with less than 1 Fano-factor as shown in Fig. 7. Such kind of spiking in LIFH neuron is possible when difference between $\lambda_{E}$ and $\lambda_{I}$ is large.

\section{Result Analysis and Conclusion}

Unlike other bio-physical neuron models, analytically solvable capability of LIF neuron is higher due to its linear representation though it is distant from a real neuron in many bio-physical mechanisms. Non-linearity in LIF neuron model arises due to threshold constraint [7]. Spiking activity of visual sensory system of many insects, fly etc., where spiking-rate is higher, cannot be captured by standard LIF neuron. Memory element of a neuron is incorporated in LIF neuron by kernel function in DDF [10-12]. We have analyzed these updated LIF model and noticed that memory functions do not affect the SPDV. Analytical calculation for SPDV of considered neuron models in subthreshold regime are found asymptotically similar and exhibiting Gaussian-distribution, i.e. from Eqns. (13), (19) and (23), it is clear that when $V \rightarrow \infty, p_{S}{ }^{G} \square p_{S}{ }^{H} \square p_{S}{ }^{L}$. This finding suggests that the LIF neuron with such kind of delay kernel functions has no effect on its stationary state probability distribution of membrane potential. Therefore, delay kernel functions, which can be extended to form the membrane potential evolution process, as a Markovian process, will exhibit Gaussian distribution for membrane potential, in subthreshold regime and one can't notice the effect of memory in SPDV of experimental data. Moreover, parameter estimation techniques which are applicable for LIF models (see [13]), can also be applied for considered models in distribution delay framework. A pioneering question in studies of such kind of stochastic problems is solve the associated first passage time (FPT) problem, i.e. to find the distribution of time interval, when the membrane potential of a neuron first time reaches to its threshold value. Analytical solution of FPT associated with the simple LIF neuron model is an unsolved problem, however in some special case, one can obtain the solution of simple LIF neuron $[10,11]$. In addition to the unsolved FPT problem associated with simple LIF neuron, FPT problem associated with LIF neuron in DDF becomes the challenges in future.

Masas [33] has demonstrated that how the network of spiking neurons attains self origination and learning form noise. DDF also provides a way capture the noise values in term of previous values of membrane potential. From simulation based study, it is notable that the spiking activity of a neuron increases in DDF due to the dependency on its previous values. The delay kernel function works as a memory function and membrane potential of a neuron attains threshold value in quicker time due to memory effect, i.e. previous values of membrane potential works as learning. Such kind of spiking activity is helpful to describe experimental and physiological data more accurately especially where higher firing rate is required. We have also noticed that hypo-exponential kernel function is more robust and reliable in term of spiking activity and neuronal information processing as compared with gamma distributed kernel function, but it is still a challenging problem to find a suitable kernel function which can replicate most of the experimental data in-vivo and in-vitro.
Lim et. al. [34] has shown the implementation of the artificial neural network (ANN) at hardware level by applying neuristor-based leaky integrate-and-fire neuron model. LIF neuron model in DDF can be an alternate model for such kind of hardware level implementation of ANN due to the increase in spiking activity.

\section{ACKNOWLEDGMENT}

The authors are extremely grateful to the reviewers whose comments have led to significant improvement in the quality of the paper.

\section{REFERENCES}

[1] P. Dayan, and L.F. Abbott "Theoretical Neuroscience: Computational and Mathematical Modeling of Neural Systems", The MIT Press, 2001.

[2] G.B. Ermentrout, and D.H. Terman, "Mathematical Foundations of Neuroscience", Springer, 2010.

[3] W. Gerstner, R. Naud, "How Good are Neuron Models?" Science, vol. 326, no, 5961, 2009, pp. 379-380.

[4] M.I. Rabinovich, P. Varona, A. I, Selverston, and H.D.I. Abarbanel, "Reviews of Modern Physics", vol. 78, no. 4, 2006, pp. 1213-1256.

[5] C. Koch, "Biophysics of Computation: Information Processing in Single Neurons", Computational Neuroscience, Oxford University Press, 1998.

[6] A.N. Burkitt, "A Review of the Integrate-and-Fire Neuron Model: I. Homogeneous Synaptic Input", Biological Cybernatics, vol. 95 no.1, 2006, 1-19.

[7] E.M. Izhikevich, "Dynamical Systems in Neuroscience: The Geometry of Excitability and Bursting", Computational Neuroscience, MIT Press, 2007.

[8] F. Gabbiani, and S.J. Cox, "Mathematics for Neuroscientists", Academic Press, 2006.

[9] C.F. Lo, and T.K. Chung, "Neural Information Processing: Lecture Notes in Computer Science", vol. 4232, 2006, pp. 324-331.

[10] Karmeshu, V. Gupta, and K.V. Kadambari, "Neuronal model with Distributed Delay: Analysis and Simulation Study for Gamma Distribution Memory Kernel”, Biological Cybernatics, vol. 104, no. 6, 2011, pp. 369383.

[11] S.K. Sharma, and Karmeshu, "Neuronal Model with Distributed Delay: Emergence of Unimodal and Bimodal ISI Distributions", IEEE Transactions on Nanobiosciences, vol. 12, no. 1, 2013, pp. 1-12.

[12] S.K. Choudhary, K. Singh, and S.K. Bharti, "Variability in spiking pattern of leaky integrate-and-fire neuron due to excitatory and inhibitory potentials", Computing for Sustainable Global Development

[13] (INDIACom), 2015, pp. 2025-2030.

[14] M. Rudolph, and A. Destexhe, "On the Use of Analytical Expressions for the Voltage Distribution to Analyze Intercellular Recordings", Neural Computation, vol. 18, 2006, pp. 2917-2922.

[15] S. Ruan, and R.S. Filfil, "Dynamics of a Two-Neuron System with Discrete and Distributed Delays", Physica D, vol. 191, 2004, 323-342.

[16] R.B. Northrop, "Introduction to Dynamic Modeling of Neuro-Sensory Systems", CRC Press, 2000.

[17] Srdjan Ostojic, "Two types of asynchronous activity in networks of excitatory and inhibitory spiking neurons", Nature Neuroscience, Vol. 17, No. 4, pp. 594-602. 2014.

[18] N. Ho, and A. Destexhe, "Synaptic Background Activity Enhances the Responsiveness of Neocortical Pyramidal Neurons", The American Physiological Society, vol. 84, no. 3, pp. 1488-1496.

[19] A. Destexhe, and D. Pare, "Impact of Network Activity on the Integrative Properties of Neocortical Pyramidal Neurons In Vivo", The American Physiological Society, vol. 81, no. 4, 1999, pp. 1531-1547.

[20] A.N. Burkitt, "A Review of the Integrate-and-Fire Neuron Model: II. Inhomogeneous Synaptic Input and Network Properties", Biological Cybernatics, vol. 95, no. 2, 2006, pp. 97-112.

[21] T.D. Frank, "Nonlinear Fokker-Planck Equations: Fundamentals and Applications", Springer Series in Synergetics, Springer, 2005.

[22] F.Y.M. Wan, and H.C. Tuckwell, "Neuronal Firing and Input Variability", Journal of Theoretical Neurobiology, vol. 1, 1982, pp. 197-218.

[23] M.P. Nawrot, C. Boucsein, V.M. Rodriguez, A. Riehle, and A.A.S. Rotter, "Measurement of Variability Dynamics in Cortical Spike Trains", Journal 
of Neuroscience Methods, vol. 169, 2008, 374-390.

[24] F. Rieke, D. Warland, R.R. Steveninck, and W. Bialek, "Spikes: Exploring The Neural Code", MIT Press, 1997.

[25] A.B. Arcas, A.L. Fairhall, and W. Bialek, "Computation in a Single Neuron: Hodgkin and Huxley Revisited", Neural Computation, vol.15, 2003, pp. 1715-1749.

[26] A. Fairhall, E.S. Brown, and A. Barreiro, "Information Theoretic Approaches to Understanding Circuit Function", Neurobiology, vol. 22, 2012, pp. 653-659.

[27] S. Haykin, J.C. Principe, T.J. Sejnowski, and J. Mcwhirter, "New Directions in Statistical Signal Processing: From Systems to Brains", MIT Press, 2007.

[28] S. Sinanovic, and D.H. Johnson, "Toward a theory of information processing", Signal Processing, vol. 87, 2007, pp. 1326-1344.

[29] W. Gerstner, A.K. Kreiter, H. Markram, and A.V.M. Herz, "Neural Code: Firing Rate and Beyond", Proceedings National Academy of Science, USA, vol. 94, 1997, 12740-12741.

[30] W. Gerstner, and W.M. Kistler, "Spiking Neuron Models: Single Neurons, Populations, Plasticity”, Cambridge University Press, 2002.

[31] U.T. Eden, and M.A. Kramer, "Drawing Inferences from Fano Factor Calculations", Journal of Neuroscience Methods, vol. 190, 2010, pp. 149152.

[32] D.J. Higham, "An Algorithmic Introduction to Numerical Simulation of Stochastic Differential Equations", SIAM Review, vol. 46, no. 3, 2001, pp. 525-546.

[33] P. Glasserman, "Monte Carlo Methods in Financial Engineering", Springer, 2004

[34] W. Maass, "Noise as a Resource for Computation and Learning in Networks of Spiking Neurons", Proceedings of the IEEE, Vol. 102, No. 5, 2014, pp. 860-880.

[35] H. Lim, V. Kornijcuk, J. Y. Seok, S. K. Kim, I. Kim, C. S. Hwang and D. S. Jeong, "Reliability of neuronal information conveyed by unreliable neuristorbased leaky integrate-and-fire neurons: a model study", Nature: Scientific Reports 5, 5, Article number: 9776, 2015.

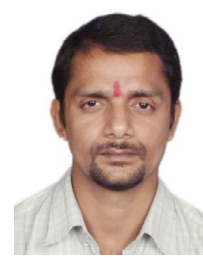

Saket Kumar Choudhary obtained his master degrees in Mathematics from the University of Allahabad, Allahabad, India in 2005, Master of Computer Application (MCA) from UPTU, Lucknow, India in 2010, Master of Technology (M.Tech) from Jawaharlal Nehru University, New Delhi, India in 2014. He is finishing his doctorate in School of Computer and Systems Sciences, Jawaharlal Nehru University, New Delhi, India. His research interest includes mathematical modeling and simulation, dynamical systems, computational neuroscience: modeling of single and coupled neurons, computer vision and digital image processing.

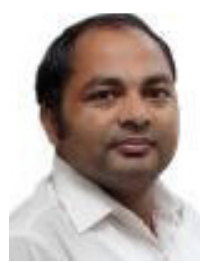

Karan Singh (M-1981) received the Engineering degree (Computer Science \& Engineering) from Kamala Nehru Institute of Technology, Sultanpur, UP, India and the M.Tech (Computer Science \& Engineering) from Motilal Nehru National Institute of Technology UP, India. He is $\mathrm{Ph} . \mathrm{D}$. (Computer Science \& Engineering) from MNNIT Allahabad deemed university. Currently, he is working in school of Computer \& Systems Sciences, Jawaharlal Nehru University, New Delhi. His primary research interests are in computer network and computer network security. He is reviewer of IEEE \& Elsevier conferences and reviewer of International journals. He is organizer of various workshop, Conference and training. Recently, Dr. Karan work as General Chair of Qshine 2013. Dr. Singh has been joined as Professional member of IEEE, ACM, CSTA, CSI, IACSIT, ICST, IAENG, ACEEE, ISOC and IEEE computer society.

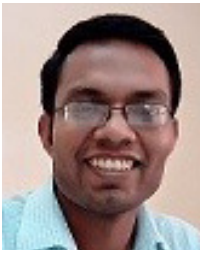

Vijender Kumar Solanki received the Master in Computer Application and Master in Engineering degree (Computer Science \& Engineering) from Maharishi Dayanand University, Rohtak, Haryana, India.(2004 \& 2007). He is pursuing Ph.D. (Computer Science \& Engineering) from Anna University, Chennai, Tamilnadu, India. His primary research interests are in Network Security, Smart Cities and Big Data. He is reviewer of IEEE, Springer \& Elsevier conferences and reviewer of International journals. He is the Guest Editor of IJRSDA, Spl Issue on "Recent Approaches on Internet of Thing, Next Generation Networks, Smart Cities and Cloud Computing" and Editor for Book on "Big Data Management and Analytics with Modern Tools \& Technologies" Published by Universities Press, India. He has delivered many Lectures in FDP, Workshop and Confrences. He can be contacted at spesinfo@yahoo.com 